\title{
Progression of large artery structural and functional alterations in Type I diabetes
}

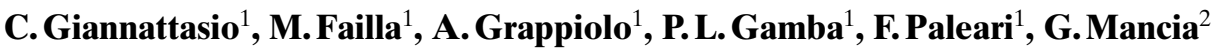 \\ ${ }^{1}$ Internal Medicine, Università of Milan-Bicocca and S.Gerardo Hospital, Monza, Milano, Italy \\ ${ }^{2}$ IRCCS, Italian Auxologic Center, Milan, Italy
}

\section{Abstract}

Aims/hypothesis. Type I (insulin-dependent) diabetes mellitus is accompanied by reduced arterial distensibility and increased arterial wall thickness even in normotensive subjects with no micro-macrovascular complications. It is not known whether, and how fast, these subclinical markers of vascular damage develop over time.

Methods. We measured arterial wall distensibility in radial, common carotid artery and abdominal aorta in 60 normotensive patients (aged $35.0 \pm 1.2$ years, means \pm SE) with Type I diabetes with no microvascular or macrovascular complications and in 20 healthy control subjects matched for age. Arterial distensibility was determined by continuous measurements of arterial diameter through echotracking techniques and by using either the Langewouters (radial artery) or the Reneman formula (carotid artery and aorta). The same echotracking techniques allowed us to ascertain the radial and carotid artery wall thickness. Data were collected before and after $23 \pm 1$ months. Results. In the first study, carotid artery distensibility was similar but radial artey and aortic distensibility was less $(p<0.01)$ in patients with diabetes than in control subjects $(-39 \%$ and $25 \%$ respectively). This was accompanied by an increase $(p<0.01)$ in both radial $(42 \%)$ and carotid artery wall thickness $(46 \%)$. After $23 \pm 1$ months diabetic subjects showed a further reduction in arterial distensibility (radial-12\%, $p<0.05$; carotid-8\%, NS; aorta-20\% $p<0.05$ ) and an increase in arterial wall thickness (radial $+15 \%$; carotid $14 \%, p<0,05)$. No change in distensibility and wall thickness values occurred in control subjects.

Conclusion/interpretation. The early reduction in arterial distensibility and increase in arterial wall thickness characterizing uncomplicated normotensive Type I diabetes patients shows a measurable worsening over the short term. [Diabetologia (2001) 44: 203-208]

Keywords Type I diabetes, arterial vessels, arterial distensibility, arterial function, atherosclerosis, arterial wall thickness, cardiovascular risk, blood pressure, follow-up.
We have previously shown that Type I (insulin-dependent) diabetes mellitus is accompanied by a reduced distensibility of the carotid artery, the radial artery and the aorta [1]. We have also shown that this

Received: 20 May 2000 and in revised form: 20 September 2000

Corresponding author: G. Mancia, Clinica Medica, Università di Milano-Bicocca, Ospedale S.Gerardo, V. Donizetti 106, Monza (MI), Italy

Abbreviations: CA, Carotid artery; AA, abdominal aorta; RA, radial artery. reduction is associated with an increased thickness of the vessel walls and that both changes can be found even in relatively young subjects with no increase in blood pressure and no macrovascular or microvascular complications [1]. This provides evidence that in diabetes there is an alteration of mechanical function and structure which involves both large elastic and middle-size muscle arteries [2-9]. It also provides evidence that this is an early phenomenon of the disease, promoting (1) the remodelling of smaller arteries $[10,11]$ which could facilitate a blood pressure increase, a condition commonly associated with diabe- 
tes [12] and (2) a greater traumatic effect in large arteries due to intravascular pressure on the vessel wall which could act as a proatherogenic factor $[13$, 14].

At present, no data exists on whether, and how fast, these abnormalities progress over time. Data like these are important, however, to establish whether diabetes-related large artery stiffening and thickening can be treated. Our study addresses this issue in patients with Type I diabetes with no hypertension and no microvascular and -macrovascular complications. This study was designed to prospectically assess the changes of distensibility and wall thickness in large and middle size arteries over a 2-year time period.

\section{Subjects and methods}

Subjects. We examined a total of 80 men and women for large artery distensibility and wall thickness twice at an interval of about $23 \pm 1$ months. A total of 60 subjects (age $35.0 \pm 1.2$ years; 32 men and 28 women) were selected who had a Type I diabetes (average duration $14.3 \pm 1.1$ years) and who had insulin treatment with no macrovascular and no microvascular (microalbuminuria, rethynopathy or neuropathy) [1]. They were also selected if they met the following criteria: (1) stable metabolic control; (2) a glycated haemoglobine less than 9; (3) no history of subclinical atherosclerotic disease; physical examination and laboratory examinations, such as a chest X-ray, resting and exercise electrocardiogram (EKG), echocardiogram and echo-Doppler of the carotid arteries, femoral arteries and abdominal aorta; (4) no blood pressure increase (systolic/diastolic values $<140 / 90 \mathrm{mmHg}$ at repeated sphygmomanometric measurements obtained at several visits); (5) no major cardiovascular disease (myocardic infarction, angina pectoris, heart failure or TIA or both, peripheral artery disease, renal insufficiency); (6) no chronic treatment with cardiovascular drugs. The remaining 20 subjects (age $34.3 \pm 1.6$ years, 12 men and 8 women) were healthy, normotensive subjects who acted as control subjects. All subjects agreed to participate in the study after being informed of its nature and purpose. The protocol of the study was approved by the ethics committee of our hospital.

Arterial distensibility and wall thickness. Arterial distensibility was measured in the radial artery, the common carotid artery and the abdominal aorta, that is in a middle-size vessel of a muscle type, in a large elastic type vessel of elastic type and in an even larger vessel with a predominant elastic respectively but also some muscle tissue. Wall thickness was measured only in the radial and common carotid artery.

Radial artery. Radial artery diameter and wall thickness were measured by an A-mode ultrasonic device (NIUS 02, Omega, Bienne Switzerland and Capital Medical Services Paris, France) 15. Briefly, a highly focalized transducer operating at a frequency of $10 \mathrm{MHz}$ was stereotaxically positioned over the radial artery $2-4 \mathrm{~cm}$ above the wrist, direct contact with the skin being prevented by use of a gel as a medium. With the subject in a supine position and the arm immobile at heart level, the transducer was oriented perpendicularly to the longitudinal axis of the vessel using the acoustic Doppler signal. After switching to A-mode, the echo beams corresponding to the posterior lumen-intima and media-adventitia interfaces were made visible on a computer screen $[15,16]$ and the wall thickness of the artery was measured [16] as the distance between the inner and outer wall echoes. The echoes scattered back from the inner posterior and anterior walls of the artery were also visualized on the computer screen and electronically digitized (using a fast transducer from analogue to digital) to allow internal diameter variations to be determined at $50 \mathrm{~Hz}$ with a spatial resolution of $0.0025 \mathrm{~mm}[15,16]$.

The device also had a photopletysmographic system (Finapres 2003, Ohmeda, Englewood, Colo., USA) which allowed blood pressure from a finger ipsilateral to the radial artery examined to be recorded at $50 \mathrm{~Hz}$ with an accuracy similar to intra-arterial blood pressure recording [17] and a resolution of $2 \mathrm{mmHg}$ [17]. The concomitant acquisition of continuous arterial diameter and blood pressure signals allowed us to calculate the diameter across the diasto-systolic pressure range. The diameter-pressure curve was analysed according to its fitting with the arctangent model of Langewouters which is based on the formula

$S=\alpha\left[\frac{\pi}{2}+\tan ^{-1}\left(\frac{P-\beta}{\gamma}\right)\right]$

where $\mathrm{S}$ is the cross sectional area, $\mathrm{P}$ is blood pressure and $\alpha, \beta$ and $\gamma$ are three optimal parameters describing the spatial position of the diameter/pressure curve [18]. Cross-sectional compliance $(\mathrm{C}=\Delta \mathrm{S} / \Delta \mathrm{P})[19]$ was calculated as:

$C=\frac{\alpha}{\gamma} \frac{1}{1+\left(\frac{P-\beta}{\gamma}\right)^{2}}$

and $\mathrm{C}$ was used to calculate cross-sectional distensibility, i.e. cross-sectional compliance divided by vessel area/pressure curve. The area under the curve was corrected for pulse pressure and used to obtain a single integrated distensibility (D) value.

Carotid artery. With the subjects in a supine position and their neck in partial extension, the diameter and wall motion of the right common carotid artery were measured $2 \mathrm{~cm}$ below the carotid bifurcation by a B-M mode echo-tracking device based on Doppler shift (Wall Track System, PIE Medical, Maastricht, Netherlands) and on a transducer operating at a frequency of $7.5 \mathrm{MHz}[20,21]$. The transducer was manually oriented perpendicularly to the longitudinal axis of the vessel under Bmode guidance. After switching to A-mode, the echoes scattered back from the anterior and posterior carotid artery walls were visualized on a screen and the corresponding radiofrequency signal was tracked by electronic tracers to allow the digitalized signal of the internal diameter variations to be determined at $50 \mathrm{~Hz}$. The spatial resolution was $300 \mu \mathrm{m}$ [22]. Blood pressure was measured from the brachial artery at the same time as ultrasound evaluation using a semiautomatic device (Dinamap 1846 SX/SXP, Critikon, Chatenay Malabry Cedex, France) and carotid artery D was derived according to the following formula [20]:

$\mathrm{D}=(2 \Delta \mathrm{D} / \mathrm{Dd}) / \Delta \mathrm{P}$

where Dd was the diastolic diameter of the vessel, $\Delta \mathrm{D}$ the systodiastolic diameter change and $\Delta \mathrm{P}$ the corresponding pulse pressure.

Carotid artery intima-media wall thickness was measured at a posterior wall site located $3 \mathrm{~cm}$ below bifurcation using an ultrasonographic device (Hewlett Packard Sonos 5500, Palo Alto, Calif., USA). Measurements were obtained by first scanning the artery in B-Mode, then freezing the digitized im- 
age in M-mode and finally tracking the inner ipoechogenic and the middle anechogenic layers 23 .

Aorta. With the subject in a lying position the diameter and wall motion of the subdiaphragmatic portion of the aorta were measured, $2-3 \mathrm{~cm}$ above the origin of the celiac plexus, by the same B-M mode echo-tracking device employed for the carotid artery $[20,21]$. The transducer (which operated at a frequency of $3.5 \mathrm{MHz}$ ) was manually oriented perpendicularly to the longitudinal axis of the vessel under B-mode guidance and brachial artery blood pressure was measured at the same time as the ultrasound evaluation using the same semiautomatic device used for the carotid artery. Aortic D was measured using the same formula used for the carotid artery. No measurement of aortic wall thickness could be made systematically for technical reasons.

All radial, carotid and aortic measurements were made by a single operator who was not aware of the experimental design and sequence (see above). The within-operator variability of radial artery, carotid artery and aortic diameter measurements at diastole (i.e. the coefficient of variation of the mean values of two measurements done at two different times ) was on average $2.5 \%, 3.5 \%$ and $4.5 \%$, respectively. The within-operator variability for radial artery and for carotid artery wall thickness were $3.0 \%$ and $4.0 \%$ respectively.

Additional measurements. Blood pressure was measured not only by the finger pressure or the Dinamap device (see above) but also by a mercury sphygmomanometer, taking the 1st and 5 th Korotkoff sounds to identify systolic and diastolic values, respectively. Heart rate was measured continuously from the finger pressure signal as the reciprocal of the pulse interval between consecutive beats. For patients with diabetes, fasting serum glucose, glycated haemoglobin, total cholesterol and creatinine values and the daily dose of insulin were found in data collected in the preceeding 3 months. Three overnight microalbuminuria measurements, an optic fundus and an electromyogram done in the preceeding 9 months were used to exclude microvascular complications.

Protocol and data analysis. Each patient was asked to come to the outpatient clinic of the San Gerardo Hospital in the afternoon following a $24 \mathrm{~h}$ period of abstinence from alcohol, caffeine consumption and cigarette smoking. The protocol of the study was as follows: (1) blood pressure was measured three times by a mercury sphygmomanometer with patients in the sitting position; (2) the subject was placed in the lying position and fitted with the finger blood pressure and the radial artery echo-tracking devices; (3) after a 10 min interval finger blood pressure, heart rate and radial artery diameter were continuously measured for $15 \mathrm{~min}$; (4) the radial artery echo-tracking device was disconnected, the semi-automatic blood pressure measuring device was placed on the brachial artery and the probe for carotid artery evaluation was placed on the neck; (5) five 6-sec acquisitions of carotid diameter throughout the cardiac cycle were obtained during a 10 min period together with semi-automatic blood pressure measurements; (6) the probe for evaluation of the abdominal aorta was positioned over the abdomen; (7) five 6-sec acquisitions of the abdominal aortic diameter throughout the cardiac cycle were obtained during a $10 \mathrm{~min}$ period together with semi-automatic blood pressure measurements done in the same way as for the carotid artery; (8) steps 1 to 7 were repeated in the second study $23 \pm 1$ months later.

The 3 sphygmomanometric blood pressure values were averaged. Radial artery diameter at diastole, D/pressure curves and $D$ values (see above) were obtained by averaging data from 5 periods of $30 \mathrm{~s}$ each, taken at 2-min intervals. Carotid artery and aortic diastolic diameter and $\mathrm{D}$ were obtained by averaging data from the five 6-sec acquisition periods. Radial artery and carotid artery wall thickness were measured on the screen image of the vessels over a 30 -sec period, based on the ultrasound image.

Results from each subject were averaged and shown as means \pm SEM separately for the control and diabetic group and the first and the second study. The two-tailed $t$ test was used to locate differences between the two studies mean values (paired $t$ test) or between the diabetic and the control group (unpaired $t$ test). The Bonferroni correction was used to account for multiple comparisons. Data were also analysed by univariate regression taking changes in arterial $\mathrm{D}$ or thickness as the dependent variables and alterations in haemodynamic or metabolic parameters as the independent ones. A $p$ value of less than 0.05 was considered statistically significant.

\section{Results}

Blood pressure, heart rate, body surface area and, for diabetic patients only, the metabolic values obtained in the first and the second study, i.e. $23 \pm 1$ months later, are shown in Table 1. Systolic blood pressure, diastolic blood pressure and heart rate were slightly lower both for the diabetic and for the control group in the second study when compared with the first study, although the differences were small and not statistically significant. In both groups, body surface area did not change over the follow-up period. In diabetic subjects, the glycated haemoglobin value and the daily dose of insulin did not change either, whereas the serum glucose value increased, the difference being statistically significant.

The arterial diameter and $\mathrm{D}$ values are shown in Table 2. At the time of the first study both control and diabetic subjects showed diameter values that increased progressively from the radial artery to the carotid artery and the aorta whereas D values were similar in the carotid artery and the aorta and less for the radial artery $(p<0.01)$. In the control group, diameter and $\mathrm{D}$ values were not different at the time of the second study. In the diabetic group diameter values were also not different in the second study when compared with the first. D values, however, were different. That is, carotid artery distensibility, which was similar to the D value of control subjects in the first study, showed a small and not significant decrease in the second study. Furthermore, radial artery and aortic $\mathrm{D}$, which were significantly lower than the values for the control group in the first study, showed a significant further decrease in the second study $(-12 \%$ and $-20 \%$, respectively).

In the control group, carotid and radial artery wall thickness did not change between the first and the second study (Table 2). In contrast, carotid and radial artery wall thicknesses were greater in the diabetic group than in the control group in the first study and showed a further significant increase $(15 \%$ and 
Table 1. Blood pressure, heart rate, body surface area and metabolic values at first and second study (i.e. after $23 \pm 1$ months) in diabetic subjects and controls.

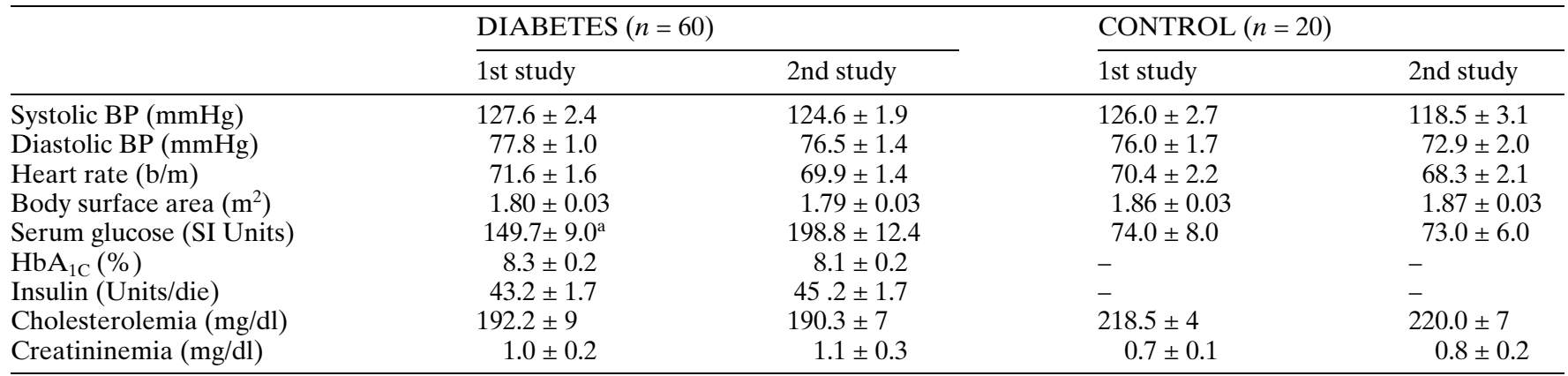

Data are showns as means \pm SE.

$p<0.01$ diabetes 1 st study vs diabetes 2 nd study

Table 2. Arterial diameter, distensibility and wall thickness in the first and second study, i. e. after $23 \pm 1$ months, in diabetic subjects and control subjects.

\begin{tabular}{|c|c|c|c|c|}
\hline & \multicolumn{2}{|c|}{ DIABETES $(n=60)$} & \multicolumn{2}{|c|}{ CONTROL $(n=20)$} \\
\hline & I st study & 2nd study & 1 st study & 2nd study \\
\hline Radial artery diameter $(\mathrm{mm})$ & $2.4 \pm 0.05$ & $2.4 \pm 0.05$ & $2.5 \pm 0.07$ & $2.5 \pm 0.09$ \\
\hline Aortic diameter $(\mathrm{mm})$ & $14.2 \pm 0.25$ & $13.8 \pm 0.33$ & $15.3 \pm 0.51$ & $15.2 \pm 0.48$ \\
\hline Radial artery distensibility $\left(1 / \mathrm{mmHg} 10^{-3}\right)$ & $0.67 \pm 0.04^{\mathrm{ab}}$ & $0.59 \pm 0.03$ & $0.92 \pm 0.07$ & $0.91 \pm 0.05$ \\
\hline Carotid artery distensibility $\left(1 / \mathrm{mmHg}^{-2} 0^{-2}\right.$ & $0.28 \pm 0.01$ & $0.26 \pm 0.01$ & $0.29 \pm 0.01$ & $0.29 \pm 0.01$ \\
\hline Aortic distensibility $\left(1 / \mathrm{mmHg} 10^{-1}\right)$ & $0.29 \pm 0.01^{\mathrm{ab}}$ & $0.21 \pm 0.01$ & $0.36 \pm 0.02$ & $0.37 \pm 0.04$ \\
\hline
\end{tabular}

Data are shown as means \pm SE.

${ }^{a} p<0.05$ diabetes 1 st study vs diabetes 2 nd study

${ }^{\mathrm{b}} p<0.01$ diabetes 1 st study vs control 1 st study

${ }^{c} p<0.01$ diabetes 1 st study vs diabetes 2 nd study

$14 \%$, respectively) in the second study. Changes in systolic blood pressure, diastolic blood pressure, heart rate, glycated haemoglobin, serum glucose and daily dose of insulin did not show any significant correlation with changes in arterial $\mathrm{D}$ values or wall thickness ( $r$ always $<0.2$, NS). This was also the case for the change in serum glucose, except for the radial artery whose D value decrease correlated with the glycaemic increase $(p<0.01)$ although the correlation coefficient was small $(r=0.32)$. There was also no correlation between changes in carotid or radial $\mathrm{D}$ and the respective changes in wall thickness ( $r$ always $<0.2, \mathrm{NS})$.

\section{Discussion}

In our relatively young patients with Type I diabetes and no microvascular or macrovascular complications, the function and structure of large arteries were already altered at baseline as shown by the reduced radial artery and aortic $\mathrm{D}$ value and the increased radial and carotid artery wall thickness when compared with control subjects matched for age.
This confirmed previous findings in which young uncomplicated diabetic patients were compared with age-matched control subjects [1]. Our study shows, however, that these values also altered after about two years, in contrast with the values of the control subjects, which did not change. Thus, the early large artery subclinical damage characteristic of Type I diabetes shows a measurable development over a relatively short time. This development involves a functional component of damage as well as a structural one.

Our study raises the question of which mechanisms are responsible for the development of the large artery stiffening and thickening seen in diabetic patients. In a previous cross-sectional study these abnormalities were variably related to blood pressure values, glycated haemoglobin and serum glucose to a limited degree [1]. In our study, however, the changes in arterial D values and wall thickness over two years showed no relation with concomitant changes in blood pressure, glycated haemoglobin and the daily dose of insulin whose average values did not increase throughout the same period. Furthermore, although serum glucose did increase over this period, this in- 
crease showed a limited relation with the change in radial artery D values and no correlation at all with changes in the aortic $\mathrm{D}$ value, the carotid $\mathrm{D}$ value and arterial thickness. This suggests that, at least within its normal range, blood pressure is not primarily responsible for arterial stiffening and thickening. It also suggests that the same is the case for a number of other endogenous and exogenous humoral factors operating in diabetes. The only partial exception is the effect of serum glucose on radial artery distensibility, possibly because an increase in serum glucose could stimulate sympathetic activity [24]. This stimulation has been shown to decrease radial artery distensibility considerably, presumably through the contraction of smooth muscle in the arterial wall [25].

Other findings of our study are firstly, in line with previous results $[1,6]$ in Type I diabetic patients, the carotid artery D value did not obviously decrease when compared with control subjects whereas a decrease in radial and aortic D value was evident. Furthermore, over the two years of the follow-up study, carotid artery D values showed a small and not consistent decrease, again at variance from radial artery and aortic D values. We cannot explain this phenomenon which seems to be at odds with the observation that carotid artery wall thickness was greater in the same diabetic patients than in control subjects in the first study and showed a further increase two years later. We speculate, however, that an increase in carotid artery wall thickness might operate (at least for a limited length of time) as a factor reducing stress on arterial walls and so preserving its mechanical response. We can also speculate that - in contrast with the radial artery and the abdominal aorta - the growth of the common carotid, an artery with a predominantly elastic structure, over the two year period involved predominantly the elastic tissue and that this had little further effect on wall D values. There is evidence from in vitro studies that diabetes exerts an influence both on cell growth and on the composition of the extracellular matrix, with an increase in its collagen component [26]. There is evidence that this could also be the case for insulin [27].

Secondly the reduction in arterial $\mathrm{D}$ values seen over two years in patients with diabetes was associated with a small and non-significant increase in vessel diameter which suggests that it was also due to an enlargement of the vessel which engaged more of its collagen components [19]. Thirdly, both the decrease in $\mathrm{D}$ values and the increase in arterial thickness were consistent and relatively large. This suggests that studies designed to determine whether therapeutic interventions can delay or prevent these alterations would be feasible if carried out for a limited period of time. Indeed, these studies might require a shorter period of time than studies aimed at determing the beneficial effects of treatment on the arterial wall abnormalities of hypertensive patients be- cause in our patients with diabetes the rate of increase of carotid artery wall thickness was $0.035 \mathrm{~mm} /$ year, i.e. almost twice as much as that reported in the general population [28]. It should be emphasized that this applies to diabetic patients free of macrovascular and microvascular complications. It is possible that the deterioration in arterial stiffening and thickening is greater in subjects who already have clinically apparent cardiovascular lesions due to diabetes.

Fourthly, the progressive reduction of arterial D values and the increase in arterial wall thickness seen in diabetic patients have clinical implications. The progressive increase in the thickness of the radial artery could reflect a progressive remodelling of small arterial vessels that - by ultimately impinging upon the lumen - could result in an increase in blood pressure, a condition more frequent in Type I diabetes than in the general population $[9,29]$. This could be further facilitated by the reduction of radial artery distensibility which might stimulate a greater secretion of endothelial substances that act as growth promoters [30]. The progressive increase in wall thickness and the reduction in arterial distensibility of larger arteries could on the other hand exert a proatherogenic influence by increasing the chance for the least perfused portion of the vessel wall (the intima) to undergo a further reduction in perfusion, thus favouring an ischemic-dependent growth of various tissue components and the traumatic effect of intravascular pressure on endothelium and other structures involved in the onset and deterioration of anatomical alterations leading to atherosclerotic lesions [31-36]. A reduction of arterial distensibility by a collar application around a large artery has been shown in animals to be accompanied by proatherogenic lesions $[13,14]$. Our finding that arterial thicknening and stiffening is greater in diabetic patients than in normal subjects even in absence of clinical complications could help clarify the mechanisms involved in diabetic macroangiopathy.

\section{References}

1. Giannattasio C, Failla M, Piperno A et al. (1999) Early impairment of alrge artery structure and function in Type I diabetes mellitus. Diabetologia 42: 987-994

2. Scarpello JH, Martin TR, Ward JD (1980) Ultrasound measurements of pulse wave velocity in the peripheral arteries of diabetics subjects. Clin Sci (Colch) 58: 533-539

3. Lo CS, Relf IR, Myers KA, Wahlqvist ML (1986) Doppler ultrasound recognition of preclinical changes in the arterial wall in diabetic subjects: compliance and pulse-wave damping. Diabetes Care 9: 27-31

4. Lehmann ED, Gosling RG, Sonksen DH (1992) Arterial wall compliance in diabetes. Diabet Med 9: 114-119

5. Lambert J, Pijpers R, Van Ittersem FJ et al. (1997) Sodium, blood pressure and arterial distensibility in insulin-dependent diabtes mellitus. Hypertension 30: 1162-1168

6. Kool MJ, Lambert J, Stehauwer CD, Hoeks AP, Struijker Bandier HA, Van Basten LM (1995) Vessel wall properties 
of large arteries in uncomplicated insulin-dependent diabetes mellitus (IDDM). Diabetes Care 18: 618-624

7. Ryden Ahlgren A, Lanne T, Wollmer P, Soressen B, Hansen F, Sundkvist G (1995) Increased arterial stiffness in women, but not in men with IDDM. Diabetologia; 38: 1082-1089

8. Christianssen T, Neubauer B (1987) Arterial wall stiffness in insulin-dependent diabetes mellitus. Acta Radiol 28: 207-209

9. Orchard TJ, Dorman JS, Maser RE et al. (1990) Prevalence of complications in IDDM by sex and duration; Pittsburgh Epidemiology of Diabetes Complications Study II. Diabetes 39: 1116-1124

10. Folkow B (1989) Resistance to blood flow at maximal vasodilatation. Hypertension 12: 635-637

11. Mulvany MJ (1995) Resistance vessel growth and remodelling: cause or consequence in cardiovascular disease. J Hum Hypertens 9: 479-485

12. Weidmann P, Beretta-Piccoli C, Trost BN (1985). Pressor factors and responsiveness in hypertension accompanying diabetes mellitus. Hypertension 7 [Suppl II]: 33-42

13. Booth RF, Martin JF, Honey AC, Hassal DG, Beesley JE, Moncada S (1993) Rapid development of atherosclerotic lesions in the rabbit carotid artery induced by perivascular manipulation. Atherosclerosis 102: 37-49

14. Marano G, Palazzesi S, Vergari A, Ferrari AU (1999) Protection by shear stress from collar-induced intimal thickening: role of nitric oxide. Arterioscler Thromb Vasc Biol 19: 2609-2614

15. Tardy Y, Meister JJ, Perret F, Brunner HR, Arditi M (1991). Noninvasive estimate of the mechanical properties of peripheral arteries from ultrasonic and photoplethysmographic measurements. Clin Phys Physiol Meas 3: 360-367

16. Girerd X, Mourad JJ, Copie X et al. (1994) Noninvasive measurement of medium-sized artery wall thickness in humans: in vitro validation. J Vasc Res 31: 114-120

17. Parati G, Casadei R, Groppelli A, Di Rienzo M, Mancia G (1989) Comparison of finger intra-arterial blood pressure monitoring at rest and during laboratory testing. Hypertension 13: 647-655

18. Langewouters GJ, Wesseling KH, Godehart WJ (1984). The static elastic properties of 45 human thoracic and 20 abdominal aortas in vitro and the parameters of a new model. J Biomech 17: 425-435

19. Dobrin PB (1983) Vascular mechanisms. In: Hamilton WF, Dow P (eds) American Physiological Society, Washington DC, pp 65-102

20. Hoeks A, Brands PJ, Smeets GA, Reneman RS (1990) Assessment of the distensibility of superficial arteries. Ultrasound Med Biol 16: 121-128

21. Kool MJ, Van Merode T, Reneman RS, Hoeks AP, Struyker Boudier H, Van Bortel LM (1994) Evaluation of reproducibility of a vessel wall movement detector system for assessment of large artery properties. Cardiovasc Res 28: 610-614
22. Arcaro G, Laurent S, Jondeau G, Hoeks A, Safar ME (1991) Stiffness of the common carotid artery in treated hypertensive patients. J Hypertens 9: 947-954

23. Salonen R, Haapdnen A, Salonen JT (1991). Measurement of intima-media thickness of common carotid arteries with high-resolution B-mode ultrasonography: inter and intraobserver variability. Ultrasound Med Biol 17: 225-230

24. Hoffman RP, Hausberg M, Sinkey CA, Anderson EA (1999) Hyperglycaemia without hyperinsulinemia produces both sympathetic neural activation and vasodilation in normal humans. J Diabetes Complications 13: 17-22

25. Failla M, Grappiolo A, Emanuelli G et al. (1999) Sympathetic tone restrains arterial distensibility in healthy and atherosclerotic subjects. J Hypertens 17: 1117-1123

26. Turk Z, Misur I, Turk N, Benko B (1999) Rat tissue collagen modified by advanced glycation: correlation with duration of diabetes and glycemic control. Clin Chem Lab Med 37: 813-820

27. Ruiz-Torres A, Melon J, Munoz FJ (1998) Insulin stimulates collagen synthesis in vascular smooth muscle cells from elderly patients. Gerontology 44: 144-148

28. Howard G, Sharrett AR, Heiss G et al. (1993) Carotid artery intimal-medial thickness distribution in general populations as evaluated by B-Mode ultrasound. Stroke 24: 1297-1304

29. Wahlqvist ML, Relf IR, Myers KA, Lo CS (1984) Diabetes and macrovascular disease: risk factors for atherogenesis and non-invasive investigation of arterial disease. Hum Nutr Clin Nutr 38: 175-184

30. Okazaki J, Comori K, Kawasaki K, Eguci D, Ishida M, Sigimaki K (1997) L-arginine inhibits smooth muscle cell proliferation of vein graft intimal thickness in hypercholesterolemic rabbits. Cardiovasc Res 36: 429-436

31. Beach KW, Brunzell JD, Conquest LL, Strandress DF (1979) The correlation of arteriosclerosis obliterans with lipoproteins in insulin-dependents and non-insulin dependent diabetes. Diabetes 28: 836-840

32. Ahlgren AR, Sundkvist G, Wollmer P, Sonesson B, Lanne $T$ (1999) Increased aortic stiffnes in woman with type 1 diabetes mellitus is associated with diabetes duration and autonomic nerve function. Diabet Med 16: 291-297

33. Kanters SD, Banga JD, Stolk RP, Algra A (1999) Incidence and determinants of mortality and cardiovascular events in diabetes mellitus: a meta-analysis. Vasc Med 4: 67-75

34. Hamalainen H, Ronnemaa T, Halonen JP, Toikka T (1999) Factors predicting lower extremities amputaions in patient with type 1 or type 2 diabetes mellitus: a population- based 7 year follow-up study. J Intern Med 246: 97-103

35. Lovestam-Adrian M, Agardh E, Agardh CD (1999) The temporal development of retinopathy and nephropaty in type 1 diabetes mellitus during 15 years diabetes duration. Diabetes Res Clin Pract 45: 15-23

36. Forrest KY, Becker DJ, Kuller LH, Wolfson SK, Orchard TJ (2000) Are predictors of coronary heart disease and lower-extremity arterial disease in type 1 diabetes the same? A prospective study. Atherosclerosis, 148: 159-169 